\title{
Incompatible double posets and double order polytopes
}

\author{
Aenne Benjes \\ Institut für Mathematik \\ Goethe-Universität \\ Frankfurt, Germany \\ aenne.benjes@stud.uni-frankfurt.de
}

Submitted: Dec 12, 2018; Accepted: Nov 14, 2019; Published: Jan 10, 2020

(C) The author. Released under the CC BY license (International 4.0).

\begin{abstract}
In 1986 Stanley associated to a poset the order polytope. The close interplay between its combinatorial and geometric properties makes the order polytope an object of tremendous interest. Double posets were introduced in 2011 by Malvenuto and Reutenauer as a generalization of Stanleys labelled posets. A double poset is a finite set equipped with two partial orders. To a double poset Chappell, Friedl and Sanyal (2017) associated the double order polytope. They determined the combinatorial structure for the class of compatible double posets. In this paper we generalize their description to all double posets and we classify the 2-level double order polytopes.
\end{abstract}

Mathematics Subject Classifications: 06A07, 52B05, 52B12, 52B20

\section{Introduction}

A partially ordered set $(P, \preceq)$, also called poset, is a finite set $P$ together with a reflexive, transitive and antisymmetric relation $\preceq$. To a poset Stanley [4] associates a convex polytope, the order polytope $\mathcal{O}(P)$, which is the set of all order-preserving functions from $P$ into the interval $[0,1]$ :

$$
\mathcal{O}(P)=\{f: P \rightarrow[0,1]: a \prec b \Rightarrow f(a) \leqslant f(b)\} .
$$

Since the order polytope reflects many combinatorial properties of the poset, it is worth to study the geometric properties of $\mathcal{O}(P)$. For more details about convex polytopes we refer to [6].

A double poset $\mathbf{P}=\left(P, \preceq_{+}, \preceq_{-}\right)$, as introduced by Malvenuto and Reutenauer [3], is a finite set $P$ together with two partial order relations $\preceq_{+}$and $\preceq_{-}$. The two underlying posets are denoted $P_{+}=\left(P, \preceq_{+}\right)$and $P_{-}=\left(P, \preceq_{-}\right)$. Chappell, Friedl, and Sanyal constructed in [2] a polytope for a double poset $\mathbf{P}$, the double order polytope given by

$$
\mathcal{O}(\mathbf{P})=\mathcal{O}\left(P, \preceq_{+}, \preceq_{-}\right):=\operatorname{conv}\left\{\left(2 \mathcal{O}\left(P_{+}\right) \times\{1\}\right) \cup\left(-2 \mathcal{O}\left(P_{-}\right) \times\{-1\}\right)\right\} \subseteq \mathbb{R}^{P} \times \mathbb{R}
$$


The interplay of the two partial orders of a double poset is reflected in the geometry of its double order polytope. The reduced double order polytope is a simpler construction that captures most properties of $\mathcal{O}(\mathbf{P})$, and is defined as

$$
\overline{\mathcal{O}}(\mathbf{P}):=\mathcal{O}(\mathbf{P}) \cap\{(f, t): t=0\}=\mathcal{O}\left(P_{+}\right)-\mathcal{O}\left(P_{-}\right) \subseteq \mathbb{R}^{P} .
$$

Note that here and in the following we write $Q-R$ for the Minkowski sum of polytopes $Q$ and $-R$. In [2, Thm 2.7] the authors gave a characterization of the facets of double order polytopes for the class of compatible double posets, that is, the case where $P_{+}$and $P_{-}$ have a common linear extension. We generalize their description to all double posets in Theorem 17. We use this description to give a complete classification of 2-level polytopes among the double order polytopes. We finish by determining the vertices of reduced double order polytopes for general double posets in Corollary 22.

\section{Double posets and double order polytopes}

Let $(P, \preceq)$ be a poset. By adjoining a new minimum $\widehat{0}$ and a new maximum $\widehat{1}$ to $P$, we obtain the poset $\widehat{P}$. The linear form associated to an order relation $a \prec b$ is the map $\ell_{a, b}: \mathbb{R}^{P} \rightarrow \mathbb{R}$ with

$$
\ell_{a, b}(f):=f(a)-f(b)
$$

for $f \in \mathbb{R}^{P}$. Moreover, for $a \in P$ we define $\ell_{a, \widehat{1}}(f):=f(a)$ and $\ell_{\widehat{0}, a}(f):=-f(a)$. With these definitions it follows that a map $f: P \rightarrow \mathbb{R}$ is contained in $\mathcal{O}(P)$ if and only if

$$
\begin{array}{ll}
\ell_{a, b}(f) \leqslant 0 & \text { for all } a \prec b, \\
\ell_{\widehat{0}, b}(f) \leqslant 0 & \text { for all } b \in P, \text { and } \\
\ell_{a, \widehat{1}}(f) \leqslant 1 & \text { for all } a \in P .
\end{array}
$$

A nonempty face of $\mathcal{O}(P)$ is a subset $F \subseteq \mathcal{O}(P)$ such that

$$
F=\mathcal{O}(P)^{\ell}:=\left\{f \in \mathcal{O}(P): \ell(f) \geqslant \ell\left(f^{\prime}\right) \text { for all } f^{\prime} \in \mathcal{O}(P)\right\}
$$

for some linear function $\ell \in\left(\mathbb{R}^{P}\right)^{*}$. If $F \neq \mathcal{O}(P)$, then $F$ is a proper face.

As mentioned before, the order polytope geometrically describes combinatorial features of the underlying poset. For example, the vertices of $\mathcal{O}(P)$ are in bijection to filters of $P$. Recall that a filter of $(P, \preceq)$ is a subset $\mathrm{J} \subseteq P$ such that $a \in \mathrm{J}$ and $a \prec b$ for $b \in P$ implies $b \in \mathrm{J}$. Dually, an ideal is a subset $\mathrm{I} \subseteq P$ such that $b \in \mathrm{I}$ and $a \prec b$ for $a \in P$ implies $a \in \mathrm{I}$.

For a combinatorial description of faces Stanley [4] introduced face partitions.

Definition 1. A (closed) face partition of a face $F \subseteq \mathcal{O}(P)$ is a partition of $\widehat{P}$ into nonempty and pairwise disjoint blocks $B_{1}, \ldots, B_{k} \subseteq \widehat{P}$ such that

$$
F=\left\{f \in \mathcal{O}(P): f \text { is constant on } B_{i} \text { for } i=1, \ldots, k\right\}
$$

and for any $i \neq j$ there is a $f \in F$ such that $f\left(B_{i}\right) \neq f\left(B_{j}\right)$. The reduced face partition of $F$ is $\mathcal{B}(F):=\left\{B_{i}:\left|B_{i}\right|>1\right\}$. 
In [2, Prop 2.1] the following description for the normal cone of an nonempty face $F \subseteq \mathcal{O}(P)$ with a reduced face partition $\mathcal{B}(F)=\left\{B_{1}, \ldots, B_{k}\right\}$ is given:

$$
N_{P}(F)=\operatorname{cone}\left\{\ell_{a, b}:[a, b] \subseteq B_{i} \text { for some } i=1, \ldots, k\right\} .
$$

We will need the following consquences that were noted in [2].

Corollary 2. Let $F \subseteq \mathcal{O}(P)$ be a nonempty face with reduced face partition $\left\{B_{1}, \ldots, B_{k}\right\}$. Then for every $\ell \in$ relint $N_{P}(F)$ and $p \in P$ the following hold:

(i) if $p \in \min \left(B_{i}\right)$ for some $i$, then $\ell_{p}>0$;

(ii) if $p \in \max \left(B_{i}\right)$ for some $i$, then $\ell_{p}<0$;

(iii) if $p \notin \bigcup_{i} B_{i}$, then $\ell_{p}=0$.

If $P$ is a polytope and $\operatorname{dim}(P)=d$, then we call the $(d-1)$-dimensional faces facets. Maximizing the linear functions $\ell(f, t)=t$ and $\ell(f, t)=-t$ over $\mathcal{O}(\mathbf{P}) \subset \mathbb{R}^{P} \times \mathbb{R}$ one obtains the facets $2 \mathcal{O}\left(P_{+}\right) \times\{1\}$ and $-2 \mathcal{O}\left(P_{-}\right) \times\{-1\}$. We call the remaining facets vertical. They are in bijection with the facets of $\overline{\mathcal{O}}(\mathbf{P})$. A facet of the reduced double order polytope is a face of the form $F=F_{+}-F_{-}$such that there is a linear function $\ell \in\left(\mathbb{R}^{P}\right)^{*}$, where $F_{+}=\mathcal{O}\left(P_{+}\right)^{\ell}$ and $F_{-}=\mathcal{O}\left(P_{-}\right)^{-\ell}$.

Definition 3. A linear function $\ell \in\left(\mathbb{R}^{P}\right)^{*}$ is called rigid for $\mathcal{O}(P)$ if it satisfies

$$
\text { relint } N_{P_{+}}\left(F_{+}\right) \cap \operatorname{relint}-N_{P_{-}}\left(F_{-}\right)=\mathbb{R}_{>0} \cdot \ell
$$

for a pair of faces $\left(F_{+}, F_{-}\right)$. Note that $F=F_{+}-F_{-}$is necessarily a facet of $\overline{\mathcal{O}}(\mathbf{P})$.

Definition 4. An alternating chain $C$ of a double poset $\mathbf{P}=\left(P, \preceq_{+}, \preceq_{-}\right)$is a finite sequence of distinct elements

$$
\widehat{0}=p_{0} \prec_{\sigma} p_{1} \prec_{-\sigma} p_{2} \prec_{\sigma} \cdots \prec_{ \pm \sigma} \quad p_{k}=\widehat{1},
$$

where $\sigma \in\{ \pm\}$. If $k$ is odd, then we additionally require that $p_{k-1} \nprec_{\sigma} p_{1}$. For an alternating chain $C$, we define a linear function $\ell_{C}$ by

$$
\ell_{C}(f):=\sigma\left(-f\left(p_{1}\right)+f\left(p_{2}\right)-\cdots+(-1)^{k-1} f\left(p_{k-1}\right)\right) .
$$

If $k=1$, then $\ell_{C} \equiv 0$. If $k>1$, then $C$ is a proper alternating chain. Let $\operatorname{sign}(C)=\tau \in$ $\{ \pm\}$ be the sign of an alternating chain $C$ if $p_{k-1} \prec_{\tau} p_{k}$ is the last relation in $C$.

Definition 5. An alternating cycle $C$ of $\mathbf{P}$ is a sequence of elements of $P$ of length $2 k$ of the form

$$
p_{0} \prec_{\sigma} \quad p_{1} \prec_{-\sigma} \quad p_{2} \prec_{\sigma} \cdots \prec_{-\sigma} \quad p_{2 k}=p_{0},
$$

where $\sigma \in\{ \pm\}$ and $p_{i} \neq p_{j}$ for $0 \leqslant i<j<2 k$. Similarly the linear function associated to $C$ is defined by

$$
\ell_{C}(f):=\sigma\left(f\left(p_{0}\right)-f\left(p_{1}\right)+f\left(p_{2}\right)-\cdots+(-1)^{2 k-1} f\left(p_{2 k-1}\right)\right) .
$$

Note that any cyclic shift yields an alternating cycle with the same linear function $\ell_{C}$. Hence, we identify an alternating cycle with all its cyclic shifts. 
Remark 6. Our definition of alternating chains differs slightly from the one given in [2] in that we require $p_{k-1} \nprec_{\sigma} p_{1}$ for a chain of odd length. Without that condition, alternating cylces would yield alternating chains with the same linear function.

The following technical fact will be of importance later.

Lemma 7. If $C$ is a proper alternating chain and $\ell_{C}$ the linear function associated to $C$, then $\max _{f \in \overline{\mathcal{O}}(\mathbf{P})} \ell_{C}(f)=1$. More precisely the following hold:

(i) if $\operatorname{sign}(C)=+$, then $\max _{f \in \mathcal{O}\left(P_{+}\right)} \ell_{C}(f)=1$ and $\min _{f \in \mathcal{O}\left(P_{-}\right)} \ell_{C}(f)=0$;

(ii) if $\operatorname{sign}(C)=-$, then $\max _{f \in \mathcal{O}\left(P_{+}\right)} \ell_{C}(f)=0$ and $\min _{f \in \mathcal{O}\left(P_{-}\right)} \ell_{C}(f)=-1$.

Proof. Since the proof works analogously, we only consider the case of an alternating chain with $\operatorname{sign}(C)=+$ and odd length:

$$
\widehat{0}=p_{0} \prec_{+} p_{1} \prec_{-} p_{2} \prec_{+} \cdots \prec_{-} p_{2 k} \prec_{+} p_{2 k+1}=\widehat{1} .
$$

The linear function $\ell_{C}$ associated to $C$ can be written in terms of the linear form of the order relation $\preceq_{+}$:

$$
\ell_{C}=\ell_{p_{0}, p_{1}}+\ell_{p_{2}, p_{3}}+\cdots+\ell_{p_{2 k}, p_{2 k+1}} .
$$

For $f \in \mathcal{O}\left(P_{+}\right)$it follows from $(1)$ that $\ell_{p_{2 i}, p_{2 i+1}}(f) \leqslant 0$ for $0 \leqslant i \leqslant k-1$ and $\ell_{p_{2 k}, \hat{1}}(f) \leqslant 1$. Hence $\ell_{C}(f) \leqslant 1$. Let $h$ be the smallest even number such that $p_{h} \prec_{+} p_{2 k}$ and let $\mathrm{J} \subseteq$ be the principal filter generated by $p_{h}$. Since $p_{2 k} \nprec+p_{1}$ we have $h \geqslant 2$ and $p_{1} \notin \mathrm{J}$. Due to the fact that $p_{2 i} \in \mathrm{J}$ implies $p_{2 i+1} \in \mathrm{J}$ it follows that $\ell_{C}\left(\mathbf{1}_{\mathrm{J}}\right)=1$, and hence $\max _{f \in \mathcal{O}\left(P_{+}\right)} \ell_{C}(f)=1$.

We can write $-\ell_{C}(f)$ in terms of the linear form of the order relation $\preceq$ - as

$$
-\ell_{C}=\ell_{p_{1}, p_{2}}+\ell_{p_{3}, p_{4}}+\cdots+\ell_{p_{2 k-1}, p_{2 k}} .
$$

For $f \in \mathcal{O}\left(P_{-}\right)$it holds that $\ell_{p_{2 i-1}, p_{2 i}}(f) \leqslant 0$ and hence $\ell_{C}(f) \geqslant 0$. Because $\ell_{C}\left(\mathbf{1}_{\varnothing}\right)=0$, $\ell_{C}$ attains this value. Hence $\max _{f \in \overline{\mathcal{O}}(\mathbf{P})} \ell_{C}(f)=1$.

Lemma 8. Let $C$ be an alternating cycle and $\ell_{C}$ the linear function associated to $C$. Then $\max _{f \in \overline{\mathcal{O}}(\mathbf{P})} \ell_{C}(f)=0$.

Proof. Let $C$ be the alternating cycle

$$
p_{0} \prec_{+} p_{1} \prec_{-} p_{2} \prec_{+} \cdots \prec_{-} p_{2 k}=p_{0} .
$$

Then we can write the linear function associated to $C$ in terms of the linear form of the order relation $\preceq_{+}$:

$$
\ell_{C}=\ell_{p_{0}, p_{1}}+\ell_{p_{2}, p_{3}}+\cdots+\ell_{p_{2 k-2}, p_{2 k-1}} .
$$

For $f \in \mathcal{O}\left(P_{+}\right)$it follows from $(1)$ that $\ell_{p_{2 i}, p_{2 i+1}}(f) \leqslant 0$ and hence $\ell_{C} \leqslant 0$. Since $\ell_{C}\left(\mathbf{1}_{\varnothing}\right)=0$ we conclude $\max _{f \in \mathcal{O}\left(P_{+}\right)} \ell_{C}(f)=0$.

Furthermore we can write $-\ell_{C}$ in terms of the linear form of the order relation $\preceq_{-}$:

$$
-\ell_{C}=\ell_{p_{1}, p_{2}}+\ell_{p_{3}, p_{4}}+\cdots+\ell_{p_{2 k-1}, p_{2 k}} .
$$

Analogously it follows $\min _{f \in \mathcal{O}\left(P_{-}\right)} \ell_{C}(f)=0$ and thus $\max _{f \in \overline{\mathcal{O}}(\mathbf{P})} \ell_{C}(f)=0$. 
The following Proposition was stated by Chappell, Friedl and Sanyal in [2].

Proposition 9. Let $\mathbf{P}=\left(P, \preceq_{+}, \preceq_{-}\right)$be a double poset. If $\ell$ is a rigid linear function for $\overline{\mathcal{O}}(\mathbf{P})$, then $\ell=\mu \ell_{C}$ for some alternating chain or alternating cycle $C$ and $\mu>0$.

Definition 10. A double poset $\mathbf{P}=\left(P, \preceq_{+}, \preceq_{-}\right)$is called compatible if $P_{+}=\left(P, \preceq_{+}\right)$ and $P_{-}=\left(P, \preceq_{-}\right)$have a common linear extension. Otherwise, $\mathbf{P}$ is incompatible.

In case $\mathbf{P}$ is a compatible double poset, it was shown in [2, Thm 2.7] that the linear functions $\ell_{C}$ associated to proper alternating chains $C$ are in bijection to rigid linear functions of $\mathcal{O}(\mathbf{P})$. Recall that a linear extension of $(P, \preceq)$ is a injective and orderpreserving map $\mathfrak{l}: P \rightarrow[n]$ where $n=|P|$.

Proposition 11. A double poset $\mathbf{P}=\left(P, \preceq_{+}, \preceq_{-}\right)$is compatible if and only if it has no alternating cycles.

Proof. If $\mathbf{P}$ is compatible, then $P_{+}$and $P_{-}$have a common linear extension $\mathfrak{l}: P \rightarrow[n]$, where $n=|P|$. Suppose there is an alternating cycle

$$
p_{0} \prec_{\sigma} p_{1} \prec_{-\sigma} p_{2} \prec_{\sigma} \cdots \prec_{-\sigma} p_{2 k}=p_{0} .
$$

Then $\mathfrak{l}$ has to satisfy

$$
\mathfrak{l}\left(p_{0}\right)<\mathfrak{l}\left(p_{1}\right)<\mathfrak{l}\left(p_{2}\right)<\cdots<\mathfrak{l}\left(p_{2 k-1}\right)<\mathfrak{l}\left(p_{2 k}\right) .
$$

Since $p_{0}=p_{2 k}$ this contradicts $\mathfrak{l}\left(p_{0}\right)<\mathfrak{l}\left(p_{2 k}\right)$.

Let $\mathbf{P}$ be a double poset without alternating cycles and $|P|=n$. Let $M=\max \left(P_{+}\right) \cap$ $\max \left(P_{-}\right)$. We claim that $M \neq \varnothing$. Otherwise, for every $p \in \max \left(P_{+}\right)$, there is a $q \in$ $P \backslash \max \left(P_{+}\right)$with $p \prec-q$. And for any such $q$ there is a $q^{\prime} \in P \backslash \max \left(P_{-}\right)$with $q \prec_{+} q^{\prime}$. Repeating yields an alternating chain or cycle. Since $|P|<\infty$ and there are no alternating cycles in $\mathbf{P}$, it has to be a finite sequence, and hence there is a $p \in P$ for which $p \in \max \left(P_{+}\right)$and $p \in \max \left(P_{-}\right)$. We can construct a map $\mathfrak{l}: P \rightarrow\{1, \ldots, n\}$ that is strictly order preserving for $\prec_{+}$and $\prec_{-}$by induction on $n$. For $n=1$, let $P=\{p\}$ and $\mathfrak{l}(p)=1$. For $n>1$, pick a $p \in M$ and define $\mathfrak{l}(p)=n$. By induction, there is a map $\mathfrak{l}: P \backslash\{p\} \rightarrow\{1, \ldots, n-1\}$ that is strictly order preserving for $\prec_{+}$and $\prec_{-}$. Any map that is constructed in this way, gives us a common linear extension for $P_{+}$and $P_{-}$and hence $\mathbf{P}$ is compatible.

The next example, taken from [2], illustrates that for incompatible double posets not every alternating chain or cycle corresponds to a facet of the double order polytope.

Example 12. Let $(P, \preceq)$ be a poset and ${ }^{o p}$ the opposite order of $\preceq$. Then $\mathbf{P}=(P, \preceq+$ ,$\left.\preceq_{-}\right)$with $\preceq_{+}=\preceq$ and $\preceq_{-}=\preceq^{o p}$ is an incompatible double poset. Since $\mathcal{O}\left(P_{+}\right)=\mathbf{1}-$ $\mathcal{O}\left(P_{-}\right)$, where $\mathbf{1}: \mathbb{R}^{P} \rightarrow \mathbb{R}$ is the function $\mathbf{1}(p)=1$ for all $p \in P$, we conclude, that the double order polytope is a prism over $\mathcal{O}\left(P_{+}\right)$. Hence the vertical facets of $\mathcal{O}(\mathbf{P})$ are prisms over the facets of $\mathcal{O}\left(P_{+}\right)$. Thus the number of facets of $\overline{\mathcal{O}}(\mathbf{P})$ equals the number of facets of $\mathcal{O}\left(P_{+}\right)$, and these are in bijection to the minima, maxima, and cover relations of $P_{+}$. For any $p \in P$ we have the alternating chains $\widehat{0} \prec_{+} p \prec_{-} \widehat{1}$ and $\widehat{0} \prec_{-} p \prec_{+} \widehat{1}$. Furthermore any cover relation $p \prec_{\sigma} q$ gives rise to the alternating cycle $p \prec_{\sigma} q \prec_{-\sigma} p$. Hence, there are more alternating chains and cycles than facets. 
In the next section, we determine the facets of the reduced double order polytope for general posets.

\section{Facets and 2-levelness}

Let $\mathbf{P}=\left(P, \preceq_{+}, \preceq_{-}\right)$be a double poset.

Definition 13. Let $\tau, \sigma \in\{ \pm\}$. An alternating chain or cycle $C$ is crossed by $a \in P$ if there are $i \neq j$ such that

$$
p_{i} \preceq_{\tau} a \prec_{\tau} p_{i+1} \text { and } p_{j} \preceq_{\sigma} a \prec_{\sigma} p_{j+1} .
$$

The motivation of this definition is the following proposition. It was shown in $[2$, Thm 2.7] that if $\mathbf{P}$ is a compatible double poset, then its alternating chains are in bijection to the facets of $\mathcal{O}(\mathbf{P})$. To prove it, a property of alternating chains of compatible double posets is used:

If $p_{i} \prec_{\sigma} p_{i+1} \prec_{-\sigma} \cdots \prec_{-\tau} p_{j} \prec_{\tau} p_{j+1}$ is part of an alternating chain $C$ with $\sigma, \tau \in\{ \pm\}$ and $i<j$, then there is no $a \in P$ such that $p_{i} \prec_{\sigma} a \prec_{\sigma} p_{i+1}$ and $p_{j} \prec_{\tau} a \prec_{\tau} p_{j+1}$. Uncrossed alternating chains and cycles of incompatible double posets fulfil this as well.

Proposition 14. If $C$ is an uncrossed alternating chain or cycle, then $\ell_{C}$ is rigid.

Proof. We only consider $C$ to be an alternating chain of the form

$$
\widehat{0}=p_{0} \prec_{+} p_{1} \prec_{-} p_{2} \prec_{+} \cdots \prec_{-} p_{2 k} \prec_{+} p_{2 k+1}=\widehat{1}
$$

since the proof works analogously for the other forms of alternating chains and cycles. Then the linear function is

$$
\ell_{C}(f)=-f\left(p_{1}\right)+f\left(p_{2}\right)-\cdots+f\left(p_{2 k}\right) .
$$

Let $F_{+}=\mathcal{O}\left(P_{+}\right)^{\ell_{C}}$ and $F_{-}=\mathcal{O}\left(P_{-}\right)^{-\ell_{C}}$ be the corresponding faces. If $\mathrm{J}$ is a filter of $P_{+}$, then $p_{2 i} \in \mathrm{J}$ implies $p_{2 i+1} \in \mathrm{J}$ for $1 \leqslant i \leqslant k$, since $p_{2 i} \prec_{+} p_{2 i+1}$. It follows from $\operatorname{sign}(C)=+$ with Lemma $7(\mathrm{i})$ that $\max _{\mathbf{J} \in P_{+}} \ell_{C}\left(\mathbf{1}_{\mathbf{J}}\right)=1$. Thus $\mathbf{1}_{\mathbf{J}} \in F_{+}$if and only if $\mathrm{J}$ does not separate $p_{2 j}$ and $p_{2 j+1}$ for $1 \leqslant j \leqslant k$, because otherwise $\ell_{C}\left(\mathbf{1}_{\jmath}\right)<1$. From Definition 1 it follows that $p_{2 j}$ and $p_{2 j+1}$ for $1 \leqslant j \leqslant k$ are not contained in different parts of the face partition $\mathcal{B}_{+}$.

If $\mathrm{J}$ is a filter of $P_{-}$, then $p_{2 i-1} \in \mathrm{J}$ implies $p_{2 i} \in \mathrm{J}$ for $1 \leqslant i \leqslant k$, since $p_{2 i-1} \prec-p_{2 i}$. It follows again with Lemma $7(\mathrm{i})$ that $\min _{\mathrm{J} \in P_{-}} \ell_{C}\left(\mathbf{1}_{\mathrm{J}}\right)=0$. Thus a filter $\mathrm{J} \subseteq P_{-}$is contained in $F_{-}$if and only if $\mathrm{J}$ does not separate $p_{2 j-1}$ and $p_{2 j}$ for $1 \leqslant j \leqslant k$, otherwise $\ell_{C}\left(\mathbf{1}_{\mathrm{J}}\right)>0$. Again from Definition 1 it follows that $p_{2 j-1}$ and $p_{2 j}$ for $1 \leqslant j \leqslant k$ are not contained in different parts of the face partition $\mathcal{B}_{-}$.

Since $C$ is an uncrossed alternating chain, there is no $a \in P$ and $i \neq j$ such that $p_{2 i} \preceq_{+}$ $a \prec_{+} p_{2 i+1}$ and $p_{2 j} \preceq_{+} a \prec_{+} p_{2 j+1}$ and hence there is $f \in F_{+}$such that $f\left(p_{2 i}\right) \neq f\left(p_{2 j}\right)$. 

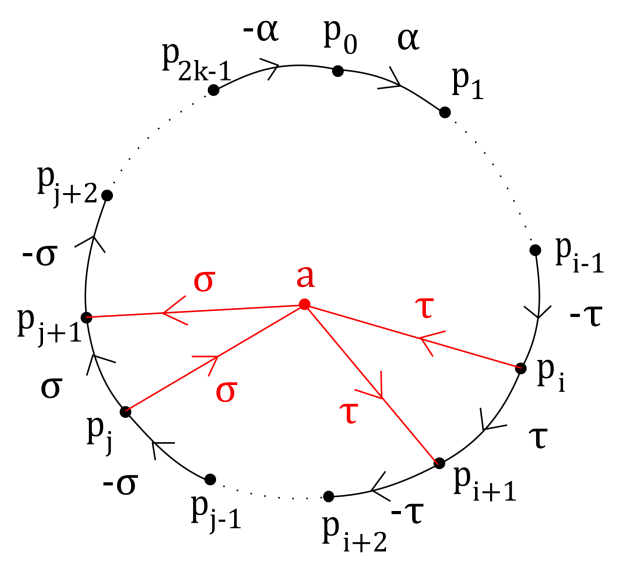

Figure 1: An alternating cycle crossed by a.

As well, there is $g \in F_{-}$such that $g\left(p_{2 i-1}\right) \neq g\left(p_{2 j-1}\right)$ for any $1 \leqslant i \leqslant j \leqslant k$. Thus, the reduced face partitions $\mathcal{B}_{ \pm}$are

$$
\begin{aligned}
& \mathcal{B}_{+}=\left\{\left[p_{0}, p_{1}\right]_{P_{+}},\left[p_{2}, p_{3}\right]_{P_{+}}, \ldots,\left[p_{2 k}, p_{2 k+1}\right]_{P_{+}}\right\} \text {and } \\
& \mathcal{B}_{-}=\left\{\left[p_{1}, p_{2}\right]_{P_{-}},\left[p_{3}, p_{4}\right]_{P_{-}}, \ldots,\left[p_{2 k-1}, p_{2 k}\right]_{P_{-}}\right\}
\end{aligned}
$$

Let $\ell$ be a linear function with $\ell(\phi)=\sum_{p \in P} \ell_{p} \phi(p)$ such that $F_{+}=\mathcal{O}\left(P_{+}\right)^{\ell}$ and $F_{-}=$ $\mathcal{O}\left(P_{-}\right)^{-\ell}$. Since for $1 \leqslant i \leqslant k$ the element $p_{2 i}$ is a minimal and $p_{2 i-1}$ is a maximal element of $\mathcal{B}_{+}$, it follows from Corollary 2 that $\ell_{p}>0$ if $p=p_{2 i-1}$ and $\ell_{p}<0$ if $p=p_{2 i}$ for $1 \leqslant i \leqslant k$. Since $C$ is an uncrossed alternating chain, it follows that if $a \in\left(p_{i}, p_{i+1}\right)_{P_{+}}$for some $i$, then $a \notin\left[p_{j}, p_{j+1}\right]_{P_{-}}$for all $j$ and vice versa. Otherwise there would be $p_{j}, p_{j+1}$ such that $p_{i} \prec_{+} a \prec_{+} p_{i+1}$ and $p_{j} \preceq_{-} a \preceq_{-} p_{j+1}$. That is why $a \notin \bigcup_{i} B_{i}$ for one of the face partitions $\mathcal{B}_{+}$or $\mathcal{B}_{-}$and hence it follows from Corollary 2(iii) that $\ell_{a}=0$. Since we assumed $F_{+}=\mathcal{O}\left(P_{+}\right)^{\ell}$ and $F_{-}=\mathcal{O}\left(P_{-}\right)^{-\ell}$, it follows that $\ell \in N_{P_{+}}\left(F_{+}\right)$and $-\ell \in N_{P_{-}}\left(F_{-}\right)$. As Equation 2 states we can write

$$
\begin{aligned}
& N_{P_{+}}\left(F_{+}\right)=\text {cone }\left\{\ell_{p_{0}, p_{1}}, \ell_{p_{2}, p_{3}}, \ldots, \ell_{p_{2 k}, p_{2 k+1}}\right\} \text { and } \\
& N_{P_{-}}\left(F_{-}\right)=\text {cone }\left\{\ell_{p_{1}, p_{2}}, \ell_{p_{3}, p_{4}}, \ldots, \ell_{p_{2 k-1}, p_{2 k}}\right\} .
\end{aligned}
$$

So $\ell \in \operatorname{relint} N_{P_{+}}\left(F_{+}\right) \cap$ relint $-N_{P_{-}}\left(F_{-}\right)$satisfies $\ell_{p_{i}}+\ell_{p_{i+1}}=0$ for all $1 \leqslant i \leqslant 2 k$ and therefore $\ell=\mu \ell_{C}$ for some $\mu>0$.

The following decomposition of crossed alternating chains and cycles will be important.

Proposition 15. Let $\mathbf{P}$ be a double poset.

(i) If $C$ is an alternating cycle crossed by a, then there are two alternating cycles $C_{1}$ and $C_{2}$ such that $\ell_{C}=\ell_{C_{1}}+\ell_{C_{2}}$.

(ii) If $C$ is an alternating chain crossed by a, then there is a proper alternating chain $C_{1}$ and an alternating cycle $C_{2}$ such that $\ell_{C}=\ell_{C_{1}}+\ell_{C_{2}}$ and $\operatorname{sign}(C)=\operatorname{sign}\left(C_{1}\right)$. 
Proof. (i) Let $C$ be a crossed alternating cycle and $i<j$ :

$$
p_{0} \prec_{+} \cdots \prec_{-\tau} p_{i} \prec_{\tau} p_{i+1} \prec_{-\tau} \cdots \prec_{-\sigma} p_{j} \prec_{\sigma} p_{j+1} \prec_{-\sigma} \cdots \prec_{-} p_{2 k}=p_{0} .
$$

(1) If $\tau=\sigma$, then

$p_{0} \prec_{+} \cdots \prec_{-\tau} p_{i} \prec_{\tau} p_{j+1} \prec_{-\tau} \cdots \prec_{-} p_{2 k}=p_{0}$ and

$p_{i+1} \prec_{-\tau} p_{i+2} \prec_{\tau} \cdots \prec_{-\tau} p_{j} \prec_{\tau} p_{i+1}$ are the two alternating cycles $C_{1}$ and $C_{2}$.

(2) If $\tau=-\sigma$, then $C_{1}$ is given by

$p_{0} \prec_{+} \cdots \prec_{-\tau} p_{i} \prec_{\tau} a \prec_{-\tau} p_{j+1} \prec_{-\tau} \cdots \prec_{+} p_{2 k}=p_{0}$ in case $p_{i} \neq a$; or

$p_{0} \prec_{+} \cdots \prec_{\tau} p_{i-1} \prec_{-\tau} p_{j+1} \prec_{\tau} \cdots \prec_{-} p_{2 k}=p_{0}$ in case $p_{i}=a$, and $C_{2}$ is given by

$p_{i} \prec_{\tau} p_{i+1} \prec_{-\tau} p_{i+2} \prec_{\tau} \cdots \prec_{\tau} p_{j} \prec_{-\tau} p_{i}$.

(ii) We only consider the case where $C$ is a crossed alternating chain starting with + and $i<j$ :

$$
\hat{0}=p_{0} \prec_{+} \cdots \prec_{-\tau} p_{i} \prec_{\tau} p_{i+1} \prec_{-\tau} \cdots \prec_{-\sigma} p_{j} \prec_{\sigma} p_{j+1} \prec_{-\sigma} \cdots \prec_{ \pm} p_{k}=\hat{1} .
$$

(3) If $\tau=\sigma$, then

$\hat{0}=p_{0} \prec_{+} \cdots \prec_{-\tau} p_{i} \prec_{\tau} p_{j+1} \prec_{-\tau} \cdots \prec_{ \pm} p_{k}=\hat{1}$ is the alternating chain $C_{1}$ and $p_{i+1} \prec_{-\tau} p_{i+2} \prec_{\tau} \cdots \prec_{-\tau} p_{j} \prec_{\tau} p_{i+1}$ is the alternating cycle $C_{2}$.

(4) If $\tau=-\sigma$, then

$\hat{0}=p_{0} \prec_{\alpha} \cdots \prec_{-\tau} p_{i} \prec_{\tau} a \prec_{-\tau} p_{j+1} \prec_{\tau} \cdots \prec_{ \pm \alpha} p_{k}=\hat{1}$ is the alternating chain $C_{1}$ in case $p_{i} \neq a$;

$\hat{0}=p_{0} \prec_{\alpha} \cdots \prec_{\tau} p_{i-1} \prec_{-\tau} p_{j+1} \prec_{\tau} \cdots \prec_{ \pm \alpha} p_{k}=\hat{1}$ is the alternating chain $C_{1}$ in case

$p_{i}=a$, and

$a \prec_{\tau} p_{i+1} \prec_{-\tau} \cdots \prec_{\tau} p_{j} \prec_{-\tau} a$ is the alternating cycle $C_{2}$ in both cases.

Corollary 16. Let $\mathbf{P}=\left(P, \preceq_{+}, \preceq_{-}\right)$be a double poset and $C$ an alternating cycle or chain. If there is an $a \in P$ such that $C$ is crossed by $a$, then $\ell_{C}$ is not rigid.

Proof. Assume that $F=\overline{\mathcal{O}}(\mathbf{P})^{\ell_{C}}$ is a facet. It follows from Proposition 15, that there are proper alternating chains or cycles $C_{1}$ and $C_{2}$ such that $\ell_{C}=\ell_{C_{1}}+\ell_{C_{2}}$ and one of the following holds:

(i) $C, C_{1}$ and $C_{2}$ are alternating cycles;

(ii) $C$ and $C_{1}$ are alternating chains that satisfy $\operatorname{sign}(C)=\operatorname{sign}\left(C_{1}\right), C_{2}$ is an alternating cycle.

Let $G=\overline{\mathcal{O}}(\mathbf{P})^{\ell_{C_{1}}}$ and $H=\overline{\mathcal{O}}(\mathbf{P})^{\ell_{C_{2}}}$ be the faces defined by $\ell_{C_{1}}$ and $\ell_{C_{2}}$. Let $f \in$ relint $F$. In case of (i), since $\ell_{C}(f)=0$ from Lemma 8 , this implies $\ell_{C_{1}}(f)=\ell_{C_{2}}(f)=0$.

In case of (ii), since $\ell_{C}(f)=1$ from Lemma 7 , this implies $\ell_{C_{1}}(f)=1$ and $\ell_{C_{2}}(f)=0$. Thus $f \in G \cap H$. Since $f$ was in relint $F$, it follows that $F \subseteq G \cap H$. The alternating chains or cycle $C_{1}$ and $C_{2}$ have a length $k>1$ and hence $\ell_{C_{i}} \neq 0$ for $i=1,2$. Thus $G, H$ are proper faces and since we have assumed that $F$ is a facet, it follows that $G$ and $H$ are facets. Since $C_{1}$ and $C_{2}$ differ by at least one element it follows, that $\ell_{C_{1}} \neq \mu \ell_{C_{2}}$ for all $\mu \in \mathbb{R}_{>0}$ and hence $G \neq H$. Thus $F$ cannot be a facet and hence $\ell_{C}$ is not rigid. 

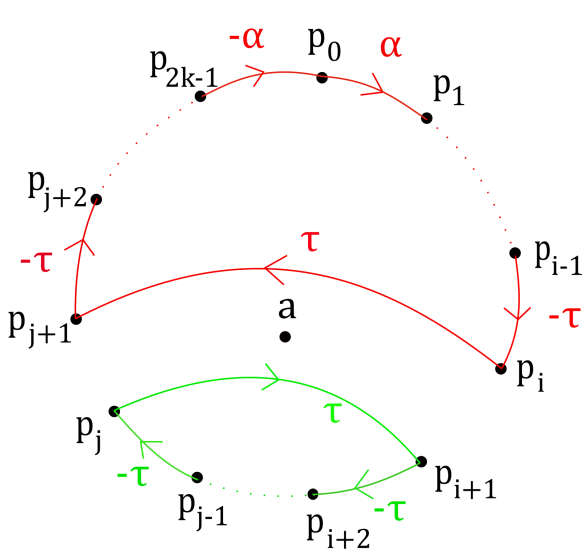

Figure 2: An alternating cycle crossed by a such that $\tau=\sigma$, and two alternating cycles $C_{1}$ (red) and $C_{2}$ (green). Those satisfy $\ell_{C}=\ell_{C_{1}}+\ell_{C_{2}}$.
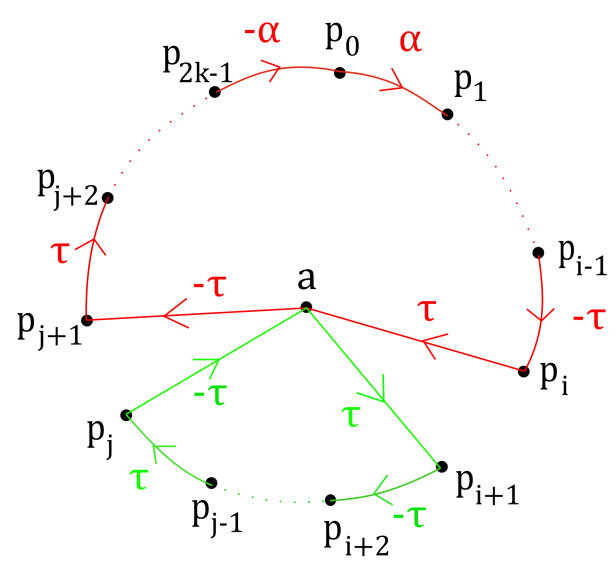

Figure 3: An alternating cycle crossed by a such that $\tau=-\sigma$, and two alternating cycles $C_{1}$ (red) and $C_{2}$ (green). Those satisfy $\ell_{C}=\ell_{C_{1}}+\ell_{C_{2}}$.

The following theorem completes the characterization of the facets of double order polytopes and follows from Proposition 14 and Corollary 16.

Theorem 17. Let $\mathbf{P}=\left(P, \preceq_{+}, \preceq_{-}\right)$be a double poset. $A$ linear function $\ell$ is rigid if and only if $\ell \in \mathbb{R}_{>0} \ell_{C}$ for some uncrossed alternating chain or cycle $C$. In particular, the facets of $\mathcal{O}(\mathbf{P})$ are in bijection to alternating chains and cycles that are not crossed by any $a \in P$.

We now turn to the question which incompatible double order polytopes are 2-level.

Definition 18. A full-dimensional polytope $Q \subseteq \mathbb{R}^{n}$ is 2-level, if for every facet-defining hyperplane $H$ there is some $t \in \mathbb{R}^{n}$ such that $H \cup(t+H)$ contains all vertices of $Q$.

2-level polytopes and compressed polytopes [5] constitute a very interesting class of polytopes in combinatorics and optimization. In particular Stanley's order polytopes are 2-level and in [2], Chappell, Friedl and Sanyal classified the 2-level polytopes among compatible double order polytopes. To include the incompatible double order polytopes we need to determine the facet-defining inequalities of $\mathcal{O}(\mathbf{P})$.

Corollary 19. Let $\mathbf{P}=\left(P, \preceq_{+}, \preceq_{-}\right)$be a double poset. Then $\mathcal{O}(\mathbf{P})$ is the set of points $(f, t) \in \mathbb{R}^{P} \times \mathbb{R}$ such that

(i) $L_{C}(f, t):=\ell_{C}(f)-\operatorname{sign}(C) t \leqslant 1$ for all uncrossed alternating chains $C$ of $P$;

(ii) $L_{C}(f, t):=\ell_{C}(f) \leqslant 0$ for all uncrossed alternating cycles of $P$.

Proof. Theorem 17 says that the facet-defining inequalities of $\mathcal{O}(\mathbf{P})$ are in bijection to the uncrossed alternating chains and cycles of $\mathbf{P}$. If $C$ is an alternating cycle and $\operatorname{sign} C=$ 
+ , then it follows by Lemma 7 that the maximal value of $\ell_{C}$ over $2 \mathcal{O}\left(P_{+}\right)$is 2 and 0 over $-2 \mathcal{O}\left(P_{-}\right)$. Since the values are exchangend for $\operatorname{sign} C=-$, the facet-defining inequalities are of the form $(i)$. If $C$ is an alternating cycle, then it follows by Lemma 8 that maximal value of $\ell_{C}$ over $2 \mathcal{O}\left(P_{+}\right)$as well as over $-2 \mathcal{O}\left(P_{-}\right)$is 0 and hence the facetdefining inequalities are of the form $(i)$.

Proposition 20. Let $\mathbf{P}=\left(P, \preceq_{+}, \preceq_{-}\right)$be a double poset and $\sigma \in\{ \pm\}$. If there are $a, b \in P$ such that $\widehat{0} \prec_{-\sigma} a \prec_{\sigma} b \prec_{-\sigma} \widehat{1}$ is an uncrossed alternating chain $C$ and it does not hold neither $a \prec_{-\sigma} b$ nor $b \prec_{-\sigma} a$, then $\mathcal{O}(\mathbf{P})$ is not 2-level.

Proof. Since $\mathcal{O}\left(P, \preceq_{+}, \preceq_{-}\right)$is 2-level if and only if $\mathcal{O}\left(P, \preceq_{-}, \preceq_{+}\right)$is 2-level we only consider $\sigma=+$.

Due to the fact that $C$ is uncrossed, the linear function $\ell_{C}$ is rigid. Then

$$
\mathrm{L}_{C}(f, t)=f(a)-f(b)+t
$$

is a facet-defining inequality of $\mathcal{O}(\mathbf{P})$. Since $b \nprec_{-} a$, there is a filter $J_{1}$ of $P_{-}$such that $b \in \mathrm{J}_{1}$ and $a \notin \mathrm{J}_{1}$. Since $a \nprec_{-} b$, there is a filter $\mathrm{J}_{2}$ of $P_{-}$such that $a \in \mathrm{J}_{2}$ and $b \notin \mathrm{J}_{2}$. As well, there is a filter $\mathrm{J}_{3}=\varnothing$ of $P_{-}$. The vertices corresponding to these three filters let $\mathrm{L}_{C}(f, t)$ take three different values:

$$
\begin{aligned}
& \mathrm{L}_{C}\left(-2 \mathrm{~J}_{1},-1\right)=0-(-2)+(-1)=1 \\
& \mathrm{~L}_{C}\left(-2 \mathrm{~J}_{2},-1\right)=-2-0+(-1)=-3 \\
& \mathrm{~L}_{C}\left(-2 \mathrm{~J}_{3},-1\right)=0-0+(-1)=-1
\end{aligned}
$$

Hence $\mathcal{O}(\mathbf{P})$ is not 2-level. For $\sigma=-$, the proof works analogously.

Theorem 21. Let $\mathbf{P}=\left(P, \preceq_{+}, \preceq_{-}\right)$be a double poset and $\sigma \in\{ \pm\}$. Then $\mathcal{O}(\mathbf{P})$ is 2-level if and only if for all $a, b \in P$ such that $a \prec_{\sigma} b$ is part of an uncrossed alternating chain or cycle it holds that $a \prec_{-\sigma} b$ or $b \prec_{-\sigma} a$.

Proof. Again, we consider only $\sigma=+$. For $\sigma=-$, the proof works analogously. If $b \prec_{-} a$, then $a \prec_{+} b$ can only be part of the alternating cycle

$$
C=a \prec_{+} b \prec_{-} a \text {. }
$$

All other alternating chains or cycles would be crossed by $a$. The corresponding linear function of the double order polytope

$$
\mathrm{L}_{C}(f, t)=f(a)-f(b)
$$

defines a facet of $\mathcal{O}(\mathbf{P})$. If $\mathrm{J}_{+}$is a filter of $P_{+}$, then $a \in \mathrm{J}_{+}$implies $b \in \mathrm{J}_{+}$and that is why $\mathrm{L}_{C}\left(2 \mathbf{1}_{\mathrm{J}_{+}}, 1\right)=0$ or $\mathrm{L}_{C}\left(2 \mathbf{1}_{\mathrm{J}_{+}}, 1\right)=-2$. If $\mathrm{J}_{-}$is a filter of $P_{-}$, then $b \in \mathrm{J}_{-}$implies $a \in \mathrm{J}_{-}$ and that is why $\mathrm{L}_{C}\left(-2 \mathbf{1}_{\mathrm{J}_{+}},-1\right)=0$ or $\mathrm{L}_{C}\left(-2 \mathbf{1}_{\mathrm{J}_{+}},-1\right)=-2$.

If $a \prec_{-} b$, then $a \prec_{+} b$ can be part of an alternating chain or cycle $C^{\prime}$ such that $C^{\prime} \neq C$. In this case all other $c \prec_{\tau} d$ in $C^{\prime}$ have to satisfy $c \prec_{-\tau} d$, where $\tau \in\{ \pm\}$. Otherwise, if 
$d \prec_{-\tau} c$, then $C^{\prime}$ would be crossed by $c$. Hence $C^{\prime}$ is an alternating chain. Let $C^{\prime}$ be the alternating chain

$$
\widehat{0}=p_{0} \prec_{\tau} p_{1} \prec_{-\tau} \cdots \prec_{ \pm} p_{k}=\widehat{1} .
$$

If $\mathrm{J}$ is a filter of $P_{+}$or $P_{-}$, then it follows from $p_{i} \in \mathrm{J}$ that $p_{i+1} \in \mathrm{J}$, since $p_{i} \prec_{+} p_{i+1}$ and $p_{i} \prec-p_{i+1}$ for $i=0, \ldots, k-1$. Let $\operatorname{sign}\left(C^{\prime}\right)=+$.

If $\mathrm{J}_{+} \subseteq P_{+}$, then $\ell_{C^{\prime}}\left(2 \mathbf{1}_{\mathrm{J}_{+}}\right)$can only take the values 2 or 0 and if $\mathrm{J}_{-} \subseteq P_{-}$, then $\ell_{C^{\prime}}\left(-2 \mathbf{1}_{J_{-}}\right)$takes the values 0 and -2 . The values are exchanged for $\operatorname{sign}\left(C^{\prime}\right)=-$. Hence

$$
\mathrm{L}_{C^{\prime}}(f, t)=\ell_{C^{\prime}}(f)-\operatorname{sign}\left(C^{\prime}\right) t,
$$

where $(f, t)$ is a vertex of $\mathcal{O}(\mathbf{P})$, attains only the values -1 and 1 . Thus $\mathcal{O}(\mathbf{P})$ is 2-level.

Assume that $\mathcal{O}(\mathbf{P})$ is 2-level. If there are $a, b \in P$ such that $a \prec_{\sigma} b$ is part of an uncrossed alternating chain or cycle and neither $a \prec_{-\sigma} b$ nor $b \prec_{-\sigma} a$, then it follows by Proposition 20 that $\mathcal{O}(\mathbf{P})$ is not 2-level.

\section{Edges of general double order polytopes}

In this last section we determine the vertical edges of double order polytopes. The edges of an order polytope $\mathcal{O}(\mathbf{P})$ were determined by Stanley [4]: Edges correspond to pairs of filters $\mathrm{J} \subset \mathrm{J}^{\prime}$ such that $\mathrm{J}^{\prime} \backslash \mathrm{J}$ is a connected poset. The vertical edges of $\mathcal{O}(\mathbf{P})$ are in bijection to the vertices of $\overline{\mathcal{O}}(\mathbf{P})$ and the following theorem shows that they also correspond to certain pairs of filters $\left(\mathrm{J}_{+}, \mathrm{J}_{-}\right)$where $\mathrm{J}_{+} \subseteq P_{+}$and $\mathrm{J}_{-} \subseteq P_{-}$.

Theorem 22. Let $\mathbf{P}=\left(P, \preceq_{+}, \preceq_{-}\right)$be a double poset and let $\mathrm{J}_{+} \subseteq P_{+}$and $\mathrm{J}_{-} \subseteq P_{-}$be filters. Let $\mathrm{I}_{+}:=P_{+} \backslash \mathrm{J}_{+}$and $\mathrm{I}_{-}:=P_{-} \backslash \mathrm{J}_{-}$be the corresponding ideals. Then $\left(2 \mathbf{1}_{\mathrm{J}_{+}}, 1\right)$ and $\left(-2 \mathbf{1}_{\mathbf{J}_{-}},-1\right)$ are the endpoints of a vertical edge of $\mathcal{O}(\mathbf{P})$ if and only if $\mathbf{1}_{\mathbf{J}_{+}}-\mathbf{1}_{\mathbf{J}_{-}}$is a vertex of $\overline{\mathcal{O}}(\mathbf{P})$ if and only if the following hold:

(i) for all $a \in \mathrm{J}_{+} \cap \mathrm{J}_{-}$there is an alternating chain

$$
\widehat{0} \prec_{-\sigma} \quad a_{1} \prec_{\sigma} \quad a_{2} \prec_{-\sigma} \cdots \prec_{ \pm} a_{k}=a \prec_{\mp} \widehat{1},
$$

where $a_{1} \in \mathrm{J}_{\sigma} \backslash \mathrm{J}_{-\sigma}$ and $a_{2}, \ldots, a_{k} \in \mathrm{J}_{+} \cap \mathrm{J}_{-}$.

(ii) for all $b \in \mathrm{I}_{+} \cap \mathrm{I}_{-}$there is an alternating chain

$$
\widehat{0} \prec_{ \pm} b=b_{1} \prec_{\mp} b_{2} \prec_{ \pm} \cdots \prec_{\sigma} \quad b_{k} \prec_{-\sigma} \widehat{1},
$$

where $b_{1}, b_{2}, \ldots, b_{k-1} \in \mathrm{I}_{+} \cap \mathrm{I}_{-}$and $b_{k} \in \mathrm{I}_{\sigma} \backslash \mathrm{I}_{-\sigma}$.

This generalizes the result of Chappell, Friedl and Sanyal in Corollary 2.17 [2], since (i) implies that $\min \mathrm{J}_{+} \cap \min \mathrm{J}_{-}=\varnothing$ and (ii) implies that $\max P_{+} \backslash \mathrm{J}_{+} \cap \max P_{-} \backslash \mathrm{J}_{-}=\varnothing$. 
Proof. From the definition of the reduced double order polytope

$$
\mathcal{O}(\mathbf{P}) \cap\{(\phi, t): t=0\}=\left(\mathcal{O}\left(P_{+}\right)-\mathcal{O}\left(P_{-}\right)\right) \times\{0\}
$$

and the fact that $\mathbf{1}_{J_{+}}-\mathbf{1}_{J_{-}}$is the midpoint between $\left(2 \mathbf{1}_{J_{+}}, 1\right)$ and $\left(-2 \mathbf{1}_{J_{-}},-1\right)$ the first equivalence follows.

To show necessity, assume that (i) is violated for some element $a \in \mathrm{J}_{+} \cap \mathrm{J}_{-}$. Let $\mathcal{C}$ be the union of all alternating chains

$$
\widehat{0} \prec_{-\sigma} \quad a_{1} \prec_{\sigma} \quad a_{2} \prec_{-\sigma} \cdots \prec_{ \pm} a_{k}=a \prec_{\mp} \widehat{1},
$$

such that $a_{1}, \ldots, a_{k} \in \mathrm{J}_{+} \cap \mathrm{J}_{-}$.

We claim that $\mathrm{J}_{+} \backslash \mathcal{C}$ is a filter in $P_{+}$. Otherwise there is an element $a_{0} \in \mathrm{J}_{+} \backslash \mathcal{C}$ and an element $a_{1} \in \mathcal{C}$ such that $a_{0} \prec_{+} a_{1}$. Since $a_{1} \in \mathcal{C}$, there is an alternating chain of the form (5). We can assume that $\sigma=-$. Otherwise, $a_{0} \prec_{+} a_{2}$ and we simply delete $a_{1}$ from the alternating chain. By construction $a_{0} \in J_{+} \backslash J_{-}$and the alternating chain $a_{0} \prec_{+} a_{1} \prec_{-} \cdots \prec_{ \pm} a_{k}=a$ contradicts our assumption.

The same argument yields that $J_{-} \backslash \mathcal{C}$ is a filter in $P$. Thus $\mathbf{1}_{J_{+}}-\mathbf{1}_{J_{-}}=\mathbf{1}_{J_{+} \backslash \mathcal{C}}-\mathbf{1}_{J_{-} \backslash \mathcal{C}}$ and therefore $\mathbf{1}_{\boldsymbol{J}_{+}}-\mathbf{1}_{J_{-}}$cannot be a vertex of $\overline{\mathcal{O}}(\mathbf{P})$. The same argument shows necessity of (ii). Indeed, let us write $P^{\text {op }}$ for the poset $P$ with the opposite order relation. Filters of $P^{\mathrm{op}}$ are ideals in $P$ and conversely and $\mathcal{O}\left(P^{\mathrm{op}}\right)=\mathbf{1}-\mathcal{O}(P)$. In particular $\mathcal{O}\left(P_{+}^{\mathrm{op}}\right)-$ $\mathcal{O}\left(P_{-}^{\text {op }}\right)=\mathcal{O}\left(P_{-}\right)-\mathcal{O}\left(P_{+}\right)=-\overline{\mathcal{O}}(\mathbf{P})$. Since $\mathbf{1}_{\mathrm{J}_{+}}-\mathbf{1}_{\mathrm{J}_{-}}$is a vertex of $\overline{\mathcal{O}}(\mathbf{P})$ if and only if $\mathbf{1}_{J_{-}}-\mathbf{1}_{J_{+}}=\mathbf{1}_{\mathbf{l}_{+}}-\mathbf{1}_{\mathrm{I}_{-}}$is a vertex of $-\overline{\mathcal{O}}(\mathbf{P})$, condition (ii) is identical to condition (i) for the opposites of $P_{+}$and $P_{-}$.

For sufficiency, let $a \in \min \mathrm{J}_{+}$. If $a \in \mathrm{J}_{+} \backslash \mathrm{J}_{-}$, then set $\ell_{+a}(f):=f(a)$. If $a \in \mathrm{J}_{+} \cap \mathrm{J}_{-}$, then let

$$
\widehat{0} \prec_{-\sigma} \quad a_{1} \prec_{\sigma} \quad a_{2} \prec_{-\sigma} \cdots \prec_{-} a_{k}=a \prec_{+} \widehat{1}
$$

be a chain $C$ as in (i). Note $\operatorname{sign}(C)=+$ since $a \in \min _{+}$. Lemma 7(i) yields that $\ell_{+a}\left(\mathbf{1}_{J_{+}^{\prime}}\right) \leqslant 1=\ell_{+a}\left(\mathbf{1}_{J_{+}}\right)$for every filter $J_{+}^{\prime} \subseteq P_{+}$. Moreover, if $\ell_{+a}\left(\mathbf{1}_{J_{+}^{\prime}}\right)=1$, then $a \in \mathrm{J}_{+}^{\prime}$. Again by Lemma $7(\mathrm{i})$, we have $\ell_{+a}\left(-\mathbf{1}_{\mathrm{J}_{-}^{\prime}}\right) \leqslant 0=\ell_{+a}\left(-\mathbf{1}_{\mathrm{J}_{-}}\right)$for all filter $\mathrm{J}_{-}^{\prime} \subseteq P_{-}$. Analogously, we use (ii) and define $\ell_{+b}$ for all $b \in \max P_{+} \backslash \mathrm{J}_{+}$. We set

$$
\ell_{+}(f):=\sum_{a \in \min J_{+}} \ell_{+a}(f)+\sum_{b \in \max P_{+} \backslash J_{+}} \ell_{+b}(f) .
$$

Then $\ell_{+}$is maximized over $\overline{\mathcal{O}}(\mathbf{P})$ at points $\mathbf{1}_{J_{+}}-\mathbf{1}_{J_{-}^{\prime}}$ for some $\mathrm{J}_{-}^{\prime} \subseteq P_{-}$. Importantly, $\mathbf{1}_{J_{+}}-\mathbf{1}_{J_{-}}$is one of the maximizers.

The same construction applied to $J_{-}$yields a function $\ell_{-}(f)$ which is maximized over $\overline{\mathcal{O}}(\mathbf{P})$ at points $\mathbf{1}_{J_{+}^{\prime}}-\mathbf{1}_{J_{-}}$for some $J_{+}^{\prime} \subseteq P_{+}$. Again, $\mathbf{1}_{J_{+}}-\mathbf{1}_{J_{-}}$is one of the maximizers. It follows that the linear function $\ell_{+}+\ell_{-}$is uniquely maximized $\mathbf{1}_{J_{+}}-\mathbf{1}_{J_{-}}$over $\overline{\mathcal{O}}(\mathbf{P})$.

\section{Acknowledgements}

This paper is based on my Bachelor thesis [1], written under the supervision of Professor Raman Sanyal. I would like to thank him for motivation and support while writing the article. 


\section{References}

[1] Aenne Benjes. A characterization of the facets of incompatible double order polytopes. Bachelor thesis, Goethe-Universität Frankfurt, 2018. 12

[2] Thomas Chappell, Tobias Friedl, and Raman Sanyal. Two double poset polytopes. SIAM J. Discrete Math., 31(4):2378-2413, 2017. 1, 2, 3, 4, 5, 6, 9, 11

[3] Claudia Malvenuto and Christophe Reutenauer. A self paired Hopf algebra on double posets and a Littlewood-Richardson rule. Journal of Combinatorial Theory Series A, 2011. 1

[4] Richard P. Stanley. Two poset polytopes. Discrete Comput. Geom., 1(1):9-23, 1986. $1,2,11$

[5] Seth Sullivant. Compressed polytopes and statistical disclosure limitation. Tohoku Math. J. (2), 58(3):433-445, 2006. 9

[6] Günter M. Ziegler. Lectures on Polytopes, volume 152 of Graduate Texts in Mathematics. Springer-Verlag, New York, 1995. 1 